\title{
Investigation of phosphoric acid and water transport in the high temperature proton exchange membrane fuel cells
}

\author{
Mu Sun ${ }^{1}$, Jicai Huang ${ }^{1}$, Zhangxun Xia ${ }^{1}$, Suli Wang ${ }^{2}$, and Gongquan Sun $^{3}$ \\ ${ }^{1}$ Dalian Institute of Chemical Physics \\ ${ }^{2}$ Chinese Academy of Sciences \\ ${ }^{3}$ Dalian Institute of Chemical Physics, Chinese Academy of Sciences
}

November 14, 2021

\begin{abstract}
A three-dimensional, non-isothermal and multiphase model of high temperature proton exchange membrane fuel cells is built to investigate water and phosphoric acid transportation, in which a spherical agglomerate model considering catalyst layer structure and liquid phase fraction is applied to determine the electrochemical kinetics in the cathode catalyst layer. Experimental polarization curve, water proportion in the anode outlet gas and phosphoric acid distribution are selected for validation. It is found that the simulated results can represent the experimental data with reasonable accuracy. Based on the model, the effects of current density and stoichiometry on the variable distributions are analyzed. The results show that water in anode is mainly from cathode by concentration diffusion of liquid water. A higher current density leads to a greater electro-migration of phosphoric acid from cathode to anode and a higher liquid phase fraction in anode,while a lower phosphoric acid concentration in the fuel cells.
\end{abstract}

\section{Hosted file}

Investigation of phosphoric acid and water transport in the high temperature proton exchange membrane $f$ available at https://authorea.com/users/446108/articles/545438-investigation-of-phosphoricacid-and-water-transport-in-the-high-temperature-proton-exchange-membrane-fuel-cells 